\title{
Factors Associated with Mortality in Patients with a Solid Malignancy Admitted to the Intensive Care Unit - A Prospective Observational Study
}

\author{
Pascal Kingah, Nasser Alzubaidi, Jihane Zaza Dit Yafawi, Emad Shehada, Khaled \\ Alshabani, Ayman O. Soubani* \\ Division of Pulmonary, Critical Care and Sleep Medicine, Wayne State University School of Medicine, 3990 John R, 3 \\ Hudson, Detroit MI 48201, USA
}

\begin{abstract}
Purpose: Several studies show conflicting results regarding the prognosis and predictors of the outcome of critically ill patients with a solid malignancy. This study aims to determine the outcome of critically ill patients, admitted to a hospital, with a solid malignancy and the factors associated with the outcomes.

Methods and Materials: All patients with a solid malignancy admitted to an intensive care unit (ICU) at a tertiary academic medical center were enrolled. Clinical data upon admission and during ICU stay were collected. Hospital, ICU, and six months outcomes were documented.

Results: There were 252 patients with a solid malignancy during the study period. Urogenital malignancies were the most common (26.3\%) followed by lung cancer $(23.5 \%)$. Acute respiratory failure was the most common ICU diagnosis (51.6\%) followed by sepsis in $46 \%$. ICU mortality and hospital mortality were $21.8 \%$ and $34.3 \%$. Six months mortality was $38.4 \%$. Using multivariate analysis, acute kidney injury, OR $2.82,95 \% \mathrm{Cl} 1.50-5.32$ and $\mathrm{P}=0.001$, use of mechanical ventilation, $\mathrm{OR} 2.6795 \% \mathrm{Cl} 1.37-5.19$ and $\mathrm{P}=0.004$ and performance status of $\geq 2$ with $\mathrm{OR}$ of $3.05,95 \%$ $\mathrm{Cl}$ of 1.5- 6.2 and $\mathrm{P}=0.002$ were associated with hospital mortality. There were no differences in outcome between African American patients (53\% of all patients) and other races.

Conclusion: This study reports encouraging survival rates in patients with a solid malignancy who are admitted to ICU. Patients with a poor baseline performance status require mechanical ventilation or develop acute renal failure have worse outcomes.
\end{abstract}

Keywords: Solid malignancy, critical care, outcome

Received: 3 August 2018 / Accepted: 30 September 2018

\section{INTRODUCTION}

Cancer remains one of the leading causes of death in the United States of America (USA). Currently, intensive care units (ICU) play a significant role in the management of cancer patients. Recent studies reveal that patients with cancer account for $15 \%$ of total admissions to an ICU [1]. However, patients with malignancies are responsible for almost half of the total ICU bed-days [2]. Despite significant advances in the treatment of cancer, patients admitted to an ICU with malignancies still have a higher mortality rate than patients without malignancies. Knowledge of the factors that influence outcomes in these patients is pivotal in their management, as it will help significantly in making appropriate triage decisions. While several studies have been published on the outcome predictors in patients with cancer in general or those with hematologic malignancies, there are only a few in those with solid malignancies and controversies remain regarding outcome-predictors in these patients (3). Besides, there are limited data from the USA and a lack of data on racial differences on these predictors. The primary aim was to identify the general characteristics and outcomes of patients with solid malignancies who are admitted to the intensive care unit (ICU) of a tertiary medical 
center in the United States. The usefulness of different critical care scoring systems as prognostic tools as well as the and identification of factors associated with poor outcomes were also evaluated.

\section{- METHODS}

This was a prospective cohort study in which patients were sequentially recruited over a two year period in an ICU of a tertiary medical center with a comprehensive cancer center, in Detroit, Michigan, USA. The Institutional Review Board (IRB) of Wayne State University in Detroit, Michigan approved the study. The principal investigator and his research associates screened all new admissions to the ICU on a daily basis. Patients were deemed eligible for enrollment in the study if they met the following inclusion criteria: they were 18 years of age or older, had an established diagnosis of malignancy that was under treatment or surveillance, and had a need for admission to the ICU due to life-threatening conditions during the study period. The exclusion criteria were: patients admitted under the surgical intensive care unit service, patients who were admitted to the ICU only for monitoring of chemotherapy, patients who were admitted for less than 24 hours and readmitted to the ICU within the study period.

Once eligible patients were identified, baseline data including demographics, a detailed description of malignancy, the reason for admission to the ICU and several clinical and laboratory parameters at the time of ICU admission. Also included at the time of ICU admission were baseline performance status using ECOG (Eastern Cooperative Oncology Group) score. Additionally, the Charlson baseline comorbidity index was calculated. Data regarding the patient's stage and type of malignancy were collected from patient notes. It was considered a "New Diagnosis" if the diagnosis was made within four weeks of admission to the ICU. The absence of evidence of an active primary malignancy was considered a case "in remission". Patients with relapsing malignancy were considered as "relapsed". The patients were then followed throughout their ICU stay to get updates on their ICU course until they were discharged from the ICU. Various scoring systems were used including the Acute Physiologic and Chronic Health Evaluation II (APACHE II), the APACHE III, the Simplified Acute Physiologic Score I, (SAPSI) SAPS II, Sepsis-related Organ Failure Assessment (SOFA), the Cancer Mortality Model (CMM), the Mortality Probability Model MPM) and the Logistic Organ Dys- function Score (LODS). The patients were followed afterwards only to determine the total duration of their hospitalization, condition at the time of discharge and any readmission to the ICU. Six months after their ICU admission, the patient's final outcome was determined. By reviewing the medical record, all other information was collected. No diagnostic interventions, including laboratory, work up, imaging studies and diagnostic procedure, were ordered for the sole purpose of the study. All diagnostic interventions were decided upon and ordered by the primary ICU team responsible for the patient, based on what they deemed clinically appropriate.

Standard descriptive statistics were used to describe the study population. Continuous variables were reported using the mean and standard deviation. Categorical variables were reported using counts and percentages. Univariate and multivariate logistic regression models, adjusting for the different confounding factors, were used to determine factors that could be associated with poor outcome. Receiver operator curves were produced for various scoring systems and the area under the curves were determined.

\section{DESULTS}

Of the 252 patients with solid malignancy enrolled in the study, $142(56.4 \%)$ with a mean age of 61.6 $\{\mathrm{SD} \pm 12.4\}$ years were female. One hundred and thirtythree (53\%) of the patients were African Americans. Urogenital cancers were the most frequently reported solid malignancies (66 patients;26.3\%) followed by lung cancer $(59 ; 23.5 \%)$ while head and neck cancers were the least reported $(26 ; 10.4 \%)$. The cancer stage was I in 5\% of patient, stage II in $6 \%$, stage III in $10 \%$ and stage IV in $59 \%$ of patients. In $20 \%$ of cases, the cancer stage was unknown at the time of admission to the ICU. Eighty-five patients (34.4\%) presented with a baseline performance status of 2. Most of the patients $(147 ; 58.3 \%)$ were in remission during their initial visit to the ICU. A significant majority of the patients (169; $67 \%$ ) were smokers, on average most patients spent 2.3 days in the hospital before ICU admission. A lactic acid level of more than 2 was reported in 141(60\%) of the patients. Major reasons for ICU admission included sepsis in 116(46\%) of patients and respiratory failure in $130 ; 51.6 \%$ of the patients. Other minor reasons are shown in Table 1. Major ICU interventions included the use of mechanical ventilation $127(50.4 \%)$ and administration of antibiotics 189 (75\%). 
Available online at: www.jccm.ro

Table 1. Baseline Characteristics on Intensive Care Unit Admission

\section{Variable}

Age (years)

Sex

Male

Female

Race

White

African American

Other

Underlying malignancy

Lung

Breast

Urogenital

Head and Neck

GI

Other

Baseline Performance Status

$\begin{array}{cc}0 & 20(8.1) \\ 1 & 56(22.3) \\ 2 & 87(34.4) \\ 3 & 74(29.5) \\ 4 & 15(5.7)\end{array}$

Disease status at admission

Remission

147(58.3)

New diagnosis

Relapse

Smoking History

Yes

No

Unknown

Days in a hospital before ICU admis-

sion

Absolute neutrophil count $<1000$

Lactic acid level $>2$

Main reasons for ICU admission

(patient may have more than one

diagnosis)

Sepsis

Respiratory failure

Pneumonia

Liver failure

Acute renal failure

Narcotic overdose

Following CPR

Cardiogenic pulmonary edema

In Receiver Operator Characteristic (ROC) analysis, an Area Under the Curve (AUC) greater than 0.7 was considered as a reasonable cutoff for predicting outcomes of the severity of illness. The SAPS III, Cancer Mortality Model, Mortality Probability Model II,
The Journal of Critical Care Medicine 2018;4(4) • 139

APACHE II and APACHEIII were scores that met this cutoff, with the APACHE III being the highest, with an AUC of 0.72. The SOFA score had an AUC of 0.64 when 5.2 was used as the cutoff, as shown in Table 2.

The mean ICU length of stay was 5.5 days. There were 55 deaths reported in the ICU with an estimated ICU mortality of $21.8 \%$. Hospital mortality was $34.3 \%$, and the 6-month mortality was $38.4 \%$ (Table 3 ). One hundred and one patients (40\%) were specified as "DoNot-Resuscitate". Of these, 52 were discharged alive from the ICU, and 29 were discharged alive from hospital. Fifty-one patients (20\%) were withdrawn from aggressive care being provided with comfort measures only while in the ICU. Four of these patients were discharged alive from hospital.

Following univariate analysis of possible variables associated with hospital mortality, ten factors were statistically significant. These factors included Lactic acid $>2 \mathrm{mg} / \mathrm{dL}$, use of mechanical ventilation, acute kidney injury, use of vasopressors, liver failure, sepsis, ICU length of stay $\geq 5$ days, ACLS in the ICU, respiratory failure and performance status $\geq 2$. All the scoring

Table 2. Severity of illness scores on admission to the ICU

\begin{tabular}{lcc} 
Severity of illness Score & $\begin{array}{c}\text { Score } \\
\text { Mean } \pm \text { SD }\end{array}$ & $\begin{array}{c}\text { ROC AUC } \\
\text { value to } \\
\text { predict } \\
\text { Mortality }\end{array}$ \\
\hline SAPS II & $42.7 \pm 13.8$ & 0.69 \\
SAPS III & $70.2 \pm 16$ & 0.71 \\
SOFA & $5.2 \pm 4$ & 0.64 \\
Cancer Mortality Model & $0.5 \pm 0.25$ & 0.72 \\
Mortality Probability Model III & $0.50 \pm 0.3$ & 0.70 \\
APACHE II & $21.4 \pm 7.5$ & 0.70 \\
APACHE III & $74.3 \pm 28.1$ & 0.72 \\
LODS & $0.2 \pm 0.2$ & 0.65 \\
Charlson Comorbidity Index>7 & $7.8 \pm 2.8$ & 0.59 \\
\hline
\end{tabular}

Table 3. ICU interventions and outcomes

\begin{tabular}{lc}
\hline ICU interventions & Number (\%) \\
Vasopressors & $53(21)$ \\
Antibiotics & $189(75)$ \\
Non-invasive ventilation & $38(15)$ \\
Mechanical Ventilation & $127(50.4)$ \\
Chemotherapy & $6(2.4)$ \\
Renal replacement therapy & $16(6.6)$ \\
Outcome data & \\
Length of stay in ICU, d & $5.5 \pm 7.4$ \\
ICU mortality & $55(21.8)$ \\
Hospital mortality & $86(34.3)$ \\
6 Month Mortality & $97(38.4)$
\end{tabular}


systems used were statistically significant except for the Charlson comorbidity index when a cutoff of $>7$ was used (Table 4). During multivariable analysis, the severity of illness scores were not used in the model to avoid collinearity. Factors that were significantly associated with hospital mortality included acute kidney injury, OR 2.97, 95\% CI 1.48-5.96 and $\mathrm{P}=0.002$, use of mechanical ventilation, OR 3.13 95\% CI $1.40-7.02$ and $\mathrm{P}=0.006$ and performance status of $\geq 2$ with $\mathrm{OR}$ of 3.54, 95\% CI of 1.64- 7.65 and $\mathrm{P}=0.001$ as seen in Table 5 . The receiver operator curves for the above factors were determined. The area under the curve (AUC) for acute kidney injury was 0.65 . For the use of mechanical ventilation the AUC was 0.63 , and for performance status, it was 0.59 .

Outcome measures were compared between African Americans and non-African Americans. ICU, hospital and six months mortality for African Americans were $21 \%, 34 \%$ and $40 \%$, respectively compared to $24 \%, 33 \%$ and $37 \%$, respectively in non-African Americans. There were no differences in factors associated with mortality between the two groups (Table 6).

\section{DISCUSSION}

In this prospective study to determine outcomes of patients with solid malignancy admitted to the ICU an ICU mortality of $21.8 \%$ and hospital mortality of $34.4 \%$ was reported. Similar results were reported in recent studies from Europe and South America [3]. In a prospective study in Brazil, Soares et al. (2010) reported an ICU mortality of $21 \%$ and hospital mortality of $30 \%$, while in France, Mokart et al. (2012) reported an ICU mortality of $32 \%$ and hospital mortality of $41 \%[4,5]$. In the latter study, a significant number of patients had a hematologic malignancy, and this was an essential difference between the two studies. Most of the patients in Mokart's study (2012) died as a result of secondary viral infections. In the study by Soares (2010), a significant number of patients had a solid malignancy with only a small fraction having a hematologic malignancy, making the results more suitable to be compared with the current study. In a recent systematic review of the literature, Puxty et al. (2014) reported the survival in solid cancer patients following ICU admission indication that ICU mortality ranged from $4.5 \%$ to $85 \%$ with

Table 4. Univariate analysis of possible predictors of hospital mortality

\begin{tabular}{|c|c|c|c|c|c|}
\hline Variable & Patient Cases & Mortality & OR & $95 \% \mathrm{Cl}$ & P-Value \\
\hline Neutrophil count $<1000$ & 22 & 12 & 1.68 & $0.69-4.05$ & 0.25 \\
\hline Lactic acid $>2$ & 131 & 53 & 1.80 & $1.05-3.08$ & 0.031 \\
\hline Mechanical Ventilation & 126 & 58 & 3.00 & $1.72-5.21$ & 0.001 \\
\hline Non-invasive ventilation & 38 & 16 & 1.49 & $0.73-3.00$ & 0.27 \\
\hline Acute kidney injury & 52 & 31 & 3.79 & $2.15-6.70$ & 0.001 \\
\hline Vasopressors & 52 & 25 & 2.10 & $1.12-3.91$ & 0.02 \\
\hline Liver failure & 13 & 10 & 4.17 & $1.40-12.4$ & 0.01 \\
\hline Sepsis & 113 & 48 & 1.96 & $1.15-3.32$ & 0.01 \\
\hline Pneumonia & 75 & 28 & 1.21 & $0.68-2.13$ & 0.50 \\
\hline APACHE $\| \geq 20$ & 148 & 65 & 3.13 & $1.74-5.64$ & $<0.0001$ \\
\hline APACHE III $\geq 75$ & 115 & 57 & 3.69 & $2.12-6.41$ & $<0.0001$ \\
\hline SAPS $\| \geq 40$ & 150 & 65 & 2.98 & $1.65-5.37$ & $<0.0001$ \\
\hline SAPS $I I I \geq 70$ & 127 & 62 & 4.06 & $2.29-7.20$ & $<0.0001$ \\
\hline SOFA score $\geq 5$ & 85 & 54 & 2.20 & $1.28-3.77$ & 0.004 \\
\hline Cancer Mortality Model $\geq 0.5$ & 125 & 64 & 3.24 & $1.86-5.63$ & $<0.0001$ \\
\hline Mortality Probability Model III $\geq 0.5$ & 115 & 56 & 3.40 & $1.96-5.90$ & $<0.0001$ \\
\hline ICU LOS $\geq 5$ days & 80 & 36 & 1.99 & $1.14-3.45$ & 0.015 \\
\hline Charlson Comorbidity & 175 & 68 & 2.09 & $1.12-3.9$ & 0.02 \\
\hline Performance Status $\geq 2$ & 173 & 70 & 2.72 & $1.43-5.16$ & 0.002 \\
\hline Renal Replacement Therapy & 16 & 7 & 1.48 & $0.53-4.14$ & 0.45 \\
\hline \multicolumn{6}{|l|}{ Disease Status } \\
\hline Remission & 144 & 47 & ---- & & \\
\hline New diagnosis & 47 & 15 & 0.97 & $0.48-1.95$ & 0.93 \\
\hline Relapse & 57 & 23 & 1.40 & $0.74-2.63$ & 0.30 \\
\hline
\end{tabular}


Available online at: www.jccm.ro

Table 5. Multivariable analysis of predictors of hospital mortality

\begin{tabular}{lccc} 
Covariate & OR & $\mathbf{9 5 \%} \mathbf{C l}$ & P-Value \\
Acute Kidney Injury & 2.82 & $1.50-5.32$ & 0.001 \\
Mechanical Ventilation & 2.67 & $1.37-5.19$ & 0.004 \\
ICU Length of Stay $\geq 5$ & 1.11 & $0.56-2.20$ & 0.76 \\
days & & & \\
Vasopressor use & 0.89 & $0.40-1.20$ & 0.79 \\
Liver failure & 2.95 & $0.91-9.58$ & 0.07 \\
Sepsis & 1.49 & $0.76-2.93$ & 0.245 \\
Performance status $\geq 2$ & 3.05 & $1.50-6.20$ & 0.002 \\
\hline
\end{tabular}

an average mortality of $31.2 \%$ [6]. The present study provides results from prospectively collected data exclusively from critically ill patients with a solid malignancy.

The improved outcome of critically ill patients with a solid malignancy is reasonably well established. , however, the primary challenge is identifying the factors that predict an outcome. Such factors may provide valuable information for patients, their families and physicians to avoid futile care and better management of resources. The current study showed that patients with performance status $\geq 2$, requiring mechanical ventilation with acute renal failure had a worse prognosis. Taccone et al. (2009) in their study carried out in 198 participating European ICUs, showed that patients with more than three organ dysfunctions resulted in high mortality (58\%) [1]. In another study, Soares et al. (2010) looked at the characteristics and outcome of cancer patients in Brazilian ICUs. They found that there is increased mortality in patients admitted for medical complications compared to patients admitted postoperatively. The presence of an active underlying malignancy in recurrence or progression, higher SOFA scores, poor performance status, the need for mechanical ventilation and the number of hospital days before ICU admission, were all predictors of a poor outcome. However, it is important to note that the majority of
The Journal of Critical Care Medicine 2018;4(4) • 141

the patients in the study had solid tumors and were admitted to the ICU postoperatively [4]. Mendoza et al. (2008) reported that the presence of metastatic disease and use of vasopressor agents were predictors of a poor outcome [7]. Puxtry et al. (2014) reported that poor functional status, invasive mechanical ventilation in addition to poor physiological scores, were associated with a poor prognosis [6].

The present study also addresses the value of the severity of illness and organ failure scores in predicting patient outcome. None of these scores (Table 2) accurately predicted the outcome of critically ill patients with a solid malignancy. Multiple studies evaluated one or more of these scores with variable results [8-12]. The current study is in accord with the overall impression that no one score system is better in predicting an outcome. Based on this conclusion, patients should not be denied admission to an ICU based on the severity of illness scores or severity of organ failure scores. Such patients should be given aggressive ICU care and then re-assessed. A better indicator of outcome, reported in some studies $[3,13]$, was the organ function status after an "ICU trial" for 3-5 days, whereas in the study by Lecuyer et al. (2007) the persistence or worsening of organ failure was stated to be a better outcome predictor [13].

In general, the outcome of cancer is worse in an African American population [14], however, the effect of race on the outcome of a critically ill patient remains controversial, with some studies suggesting worse outcomes in African Americans [15-17].

An important finding of the present study is that the short-term mortality of critically ill patients with a solid malignancy was not different between African Americans and other races.

The study has many strengths, being prospective with a relatively large number of enrolled diverse racial patients, reporting on only solid malignancies with

Table 6. Predictors of Hospital Mortality associated with Ethnicity

\begin{tabular}{|c|c|c|c|c|c|c|}
\hline Covariate & $\begin{array}{c}\text { OR for African } \\
\text { Americans }\end{array}$ & $95 \% \mathrm{Cl}$ & P-Value & $\begin{array}{c}\text { OR for } \\
\text { Non-African } \\
\text { Americans }\end{array}$ & $95 \% \mathrm{Cl}$ & P- Value \\
\hline Acute Kidney Injury & 1.21 & $0.70-1.99$ & 0.90 & 0.52 & $0.14-1.99$ & 0.35 \\
\hline Mechanical Ventilation & 0.88 & $0.52-1.48$ & 0.61 & 0.69 & $0.24-1.69$ & 0.50 \\
\hline ICU Length of Stay $\geq 5$ days & 0.70 & $0.40-1.21$ & 0.20 & 0.76 & $0.24-2.37$ & 0.64 \\
\hline Vasopressor use & 0.83 & $0.44-1.58$ & 0.58 & 1.14 & $0.33-3.89$ & 0.83 \\
\hline Liver failure & 0.76 & $0.24-2.42$ & 0.64 & 1.07 & $0.12-0.14$ & 0.95 \\
\hline Sepsis & 0.71 & $0.42-1.20$ & 0.20 & 0.42 & $0.13-1.29$ & 0.13 \\
\hline Performance status $\geq 2$ & 1.30 & $0.74-2.27$ & 0.36 & 1.50 & $0.45-5.00$ & 0.51 \\
\hline
\end{tabular}


purely medical indications for ICU admission. However, there are a few limitations including being a single center experience and the lack of comparison of outcomes in cancer-patients with non-cancer patients. It would be helpful to study changes in severity of illness scores after patients' admission to the ICU and determine whether these can provide better outcomepredictions. Also, the study does not provide predictors of long-term outcomes in these patients. Further prospective multicenter studies are still needed to validate the current data.

\section{- CONCLUSIONS}

The current study shows encouraging ICU, hospital and six months survival rates in patients with solid malignancies who are admitted to an ICU. There were no differences in outcome between races. The need for mechanical ventilation, acute renal failure and poor performance status were associated with worse outcomes.

\section{- CONFLICT OF INTEREST}

None to declare

\section{REFERENCES}

1. Taccone FS, Artigas AA, Sprung CL, Moreno R, Sakr Y, Vincent JL. Characteristics and outcomes of cancer patients in European ICUs. Crit Care. 2009;13(1):R15.

2. Soares M, Salluh JIF, Torres VBL, Leal JVR, Spector N. Short- and long-term outcomes of critically ill patients with cancer and prolonged ICU length of stay. Chest. 2008;134(3):520-6.

3. Soubani AO. Critical Care Prognosis and Outcomes in Patients with Cancer. Clin Chest Med. 2017;38(2):333-53

4. Soares M, Caruso P, Silva E, et al. Characteristics and outcomes of patients with cancer requiring admission to intensive care units: a prospective multicenter study. Crit Care Med. 2010;38(1):9-15.
5. Mokart D, Etienne A, Esterni B, et al. Critically ill cancer patients in the intensive care unit: short-term outcome and 1-year mortality. Acta Anaesthesiol Scand. 2012;56(2):178-89.

6. Puxty K, McLoone P, Quasim T, Kinsella J, Morrison D. Survival in solid cancer patients following intensive care unit admission. Intensive Care Med. 2014;40(10):1409-28.

7. Mendoza V, Lee A, Marik PE. The hospital-survival and prognostic factors of patients with solid tumors admitted to an ICU. Am J Hosp Palliat Care. 2008;25(3):240-3.

8. Namendys-Silva SA, Texcocano-Becerra J, Herrera-Gómez A. Application of the Sequential Organ Failure Assessment (SOFA) score to patients with cancer admitted to the intensive care unit. Am J Hosp Palliat Care. 2009;26(5):341-6.

9. Soares M, Fontes F, Dantas J, et al. Performance of six severityof-illness scores in cancer patients requiring admission to the intensive care unit: a prospective observational study. Crit Care. 2004;8(4):R194-203.

10. Unseld S, Schuepbach RA, Maggiorini M. ICU, hospital and oneyear mortality of patients suffering from solid or haematological malignancies. Swiss Med Wkly. 2013;143:w13741.

11. Kopterides P, Liberopoulos P, Ilias I, et al. General prognostic scores in outcome prediction for cancer patients admitted to the intensive care unit. Am J Crit Care. 2011;20(1):56-66.

12. Schellongowski $P$, Benesch $M$, Lang $T$, et al. Comparison of three severity scores for critically ill cancer patients. Intensive Care Med. 2004;30(3):430-6.

13. Lecuyer L, Chevret S, Thiery G, Darmon M, Schlemmer B, Azoulay E. The ICU trial: a new admission policy for cancer patients requiring mechanical ventilation. Crit Care Med. 2007;35(3):808-14.

14. DeSantis CE, Siegel RL, Sauer A, et al. Cancer statistics for African Americans, 2016: Progress and opportunities in reducing racial disparities. CA Cancer J Clin. 2016;66: 290-308

15. Khan BA, Perkins A, Hui SL, et al. Relationship Between African-American Race and Delirium in the ICU. Crit Care Med. 2016;44(9):1727-34

16. Erickson SE, Vasilevskis EE, Kuzniewicz MW, et al.. The effect of race and ethnicity on outcomes among patients in the intensive care unit: a comprehensive study involving socioeconomic status and resuscitation preferences. Crit Care Med. 2011;39(3):429-35

17. Williams JF, Zimmerman JE, Wagner DP, Hawkins M, Knaus WA. African American and white patients admitted to the intensive care unit: is there a difference in therapy and outcome? Crit Care Med. 1995;23(4):626-36 